\title{
Perceptual Functions of Perirhinal Cortex in Rats: Zero-Delay Object Recognition and Simultaneous Oddity Discriminations
}

\author{
Susan J. Bartko, ${ }^{1}$ Boyer D. Winters, ${ }^{1}$ Rosemary A. Cowell, ${ }^{2}$ Lisa M. Saksida, ${ }^{1,3}$ and Timothy J. Bussey ${ }^{1,3}$ \\ ${ }^{1}$ Department of Experimental Psychology, University of Cambridge, Cambridge CB2 3EB, United Kingdom, ${ }^{2}$ Laboratoire d'Etude de l'Apprentissage et du \\ Developpement-Centre National de la Recherche Scientifique, Université de Bourgogne, 21065 Dijon, France, and ${ }^{3}$ Medical Research Council and Wellcome \\ Trust Behavioural and Clinical Neuroscience Institute, University of Cambridge, Cambridge CB2 3EB, United Kingdom
}

The perirhinal cortex (PRh) is widely accepted as having an important role in object recognition memory in humans and animals. Contrary to claims that PRh mediates declarative memory exclusively, previous evidence suggests that PRh has a role in the perceptual processing of complex objects. In the present study, we conducted an examination of the possible role of PRh in perceptual function in rats. We examined whether bilateral excitotoxic lesions of PRh or PPRh (perirhinal plus postrhinal cortices) in the rat would cause deficits in a zero-delay object-recognition task and a simultaneous oddity discrimination task. Both of these tasks measured spontaneous (untrained, unrewarded) behavior, and the stimuli in these experiments were manipulated to produce varying levels of perceptual difficulty. As predicted by simulations using a computational model, rats with PPRh lesions were impaired in object recognition when the stimuli to be discriminated were manipulated to share many features in common. Furthermore, rats with PPRh and PRh lesions were impaired in a simultaneous oddity discrimination task when the stimuli to be discriminated were manipulated explicitly to be more perceptually similar. These findings provide support for the idea that PRh in the rat is important for the perceptual processing of complex objects, in addition to its well established role in memory.

Key words: feature ambiguity; medial temporal lobe; spontaneous object recognition; amnesia; PMFC model; ventral visual stream

\section{Introduction}

Electrophysiological data (Xiang and Brown, 1998; Brown and Aggleton, 2001), cases of medial temporal lobe (MTL) amnesia (Buffalo et al., 1998), neuroimaging studies (Pihlajamaki et al., 2004), and animal lesion studies (Meunier et al., 1993; Suzuki et al., 1993; Mumby and Pinel, 1994; Aggleton et al., 1997; Winters et al., 2004) provide converging evidence that the perirhinal cortex $(\mathrm{PRh})$ is important for object recognition memory. Although the role of the PRh in recognition memory is well established, it has previously been suggested that the PRh is also involved in perception (Eacott et al., 1994; Buckley and Gaffan, 1998; Murray and Bussey, 1999; Bussey and Saksida, 2002; Bussey et al., 2003; Norman and Eacott, 2004; Lee et al., 2006b). Others hold that PRh mediates declarative memory exclusively and is not important for object perception; impairments in performance on perceptual tasks resulting from PRh lesions are often attributed to TE damage (Buffalo et al., 1999; Stark and Squire, 2000; Levy et al., 2005; Shrager et al., 2006) and are also sometimes viewed as

\footnotetext{
Received Nov. 29, 2006; revised Jan. 31, 2007; accepted Feb. 1, 2007.

This work was supported by a Wellcome Trust Project Grant to T.J.B. and L.M.S. and a Biotechnology and Biological Sciences Research Council Grant to T.J.B., L.M.S., and B.D.W. R.A.C. was additionally supported by European Commission Sixth Framework New and Emerging Science and Technology Grant 516542. S.J.B. was additionally supported by a Ruth L. Kirschstein Predoctoral Fellowship from the National Institute of Mental Health. Correspondence should be addressed to Susan J. Bartko at the above address. E-mail: sjb237@cam.ac.uk. D0I:10.1523/JNEUROSC1.5171-06.2007

Copyright $\odot 2007$ Society for Neuroscience $\quad$ 0270-6474/07/272548-12\$15.00/0
}

mnemonic deficits, for example, if the task involves pretraining, the acquisition of a rule, or requires information to be remembered over a short delay (Buffalo et al., 1998, 1999, 2000; Holdstock et al., 2000; Hampton, 2005; Levy et al., 2005; Shrager et al., 2006; Squire et al., 2006).

The current series of experiments examined whether PRh lesions in the rat would cause deficits in object recognition and in an oddity discrimination task when objects were manipulated to produce pairs of stimuli with varying levels of perceptual similarity. These tasks were designed specifically to minimize factors such as pretraining, rule learning, and memory retention. Furthermore, across the series of experiments, we developed increasingly specific excitotoxic lesions restricted to the $\mathrm{PRh}$, which produced no damage to area TE.

In experiment 1 , simulations using a computational model (Cowell et al., 2006) are provided to make explicit our predictions and the reasons for them. In experiment 2, we examined the behavior of rats with PPRh (perirhinal plus postrhinal cortex) lesions in a modified spontaneous object recognition paradigm (Ennaceur and Delacour, 1988). This modified paradigm allowed us to test under conditions of zero delay, as the zero-delay condition in object recognition experiments is usually considered to have little or no memory load and has been used as an assay of perceptual function (Eacott et al., 1994; Buffalo et al., 1999, 2000; Holdstock et al., 2000; Levy et al., 2005). Finally, having established sets of stimuli with different levels of perceptual difficulty 
using a simple visual discrimination task (experiment 3), we then used these stimuli to examine the performance of PPRh- and PRh-lesioned rats in a novel simultaneous oddity discrimination task (experiment 4). During the oddity task, stimuli were presented to the rat simultaneously, so that stimuli were always present and there was no requirement to remember the stimuli across a delay. Performance was measured in four different perceptual conditions (low, medium, medium-high, and high).

\section{Materials and Methods Experiment 1}

It has been shown that PRh lesions can impair two-choice visual discrimination tasks when there is sufficient perceptual overlap between the stimuli (Bussey et al., 2003). This result was predicted from the theory that $\mathrm{PRh}$ contains conjunctive representations of complex visual stimuli (Bussey and Saksida, 2002). To illustrate how this putative property of PRh leads to this prediction, Bussey et al. (2003) included simulations using the connectionist "perceptual-mnemonic feature conjunction" (PMFC) model of the PRh (Bussey and Saksida, 2002) to make these predictions explicit. The pattern of data generated by the simulations matched those from the monkey experiments.

Although these experiments and simulations addressed effects of PRh lesions on visual discriminations specifically, the same predictions should hold for the canonical test of PRh function, object recognition. One such prediction is that, just as in the case of visual discriminations, PRh damage should lead to impairments in object recognition when there is sufficient perceptual overlap between the stimuli. Because, according to the PMFC model, this is a perceptual rather than a mnemonic effect, such effects should be seen even when mnemonic factors are minimized or even absent, that is, under conditions of zero delay or simultaneous presentation of sample and choice.

Cowell et al. (2006) have previously presented a connectionist model, based on the same fundamental principles as the PMFC model, that accounts for effects of PRh lesions on tests of object recognition, including object recognition with variable delays and list lengths, and with trial-unique versus repeating stimuli. In the present experiment, we present simulations using this connectionist model to make explicit the prediction that PRh lesions can impair object recognition at zero delay, when stimuli are perceptually similar. The simulations show how perceptual similarity between objects can be resolved through the use of complex conjunctive representations in PRh.

\section{Architecture of the model}

This section provides a brief overview of the connectionist network (Figure 1A) (for details, see Cowell et al., 2006). The model assumes that regions of the ventral visual stream, including the $\mathrm{PRh}$, contain visual representations that are organized hierarchically, with simple features being housed in caudal regions of the ventral visual stream, and representations of the conjunctions of those features residing in more rostral regions (Bussey and Saksida, 2002). In the connectionist network, this system of representations is reduced to a two-stage scheme, in which the first layer corresponds to a caudal region of the ventral visual stream, and the second layer to the PRh.

The caudal layer of the model combines two stimulus dimensions into a single representation; each two-dimensional combination corresponds to a visual "feature" of an object. The PRh layer combines eight stimulus dimensions into a single representation, forming a unique and fully specified representation of a visual object possessing four features. Both layers of the model are implemented using Kohonen grids. The caudal layer comprises four Kohonen grids, each of which receives two-dimensional inputs, and the $\mathrm{PRh}$ layer comprises one Kohonen grid receiving an eight-dimensional input. Thus, the PRh layer contains conjunctive representations of those visual features that are represented individually in the more caudal layer.

Kohonen grids are designed to model cortex, including computational abstractions of cortical mechanisms such as lateral inhibition; this type of network is therefore appropriate for the current investigation. Each Kohonen grid comprises a two-dimensional array of processing units that
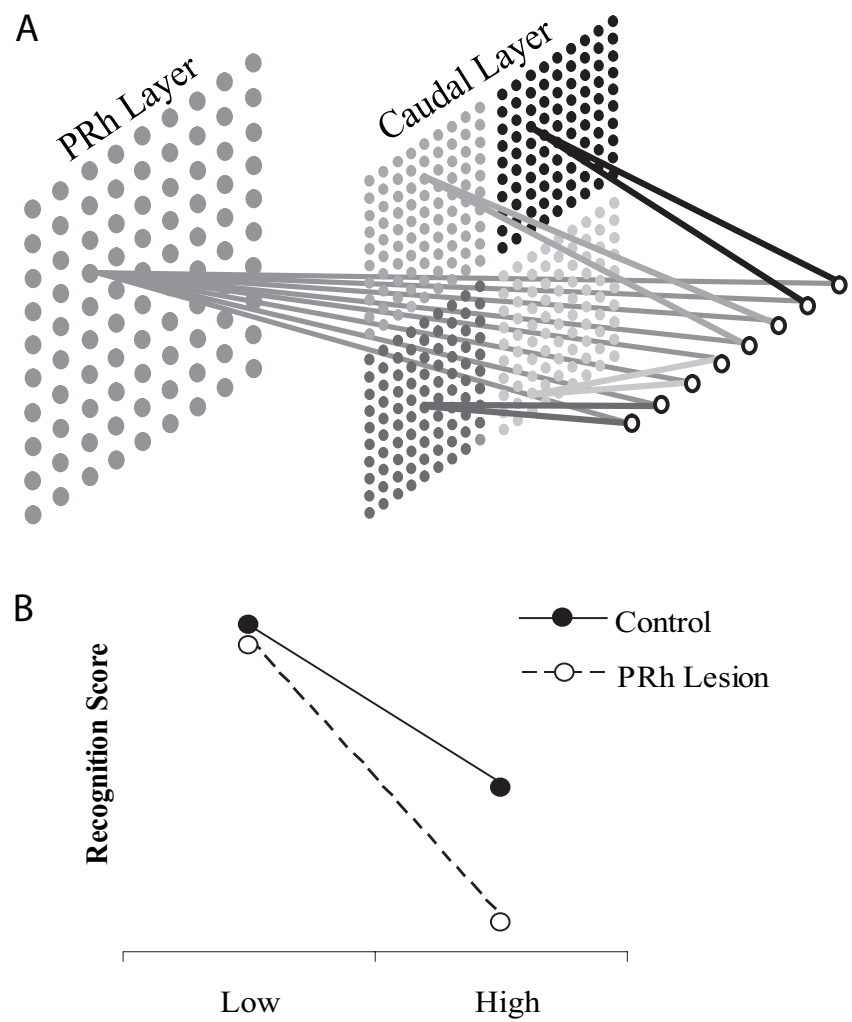

Stimulus Condition

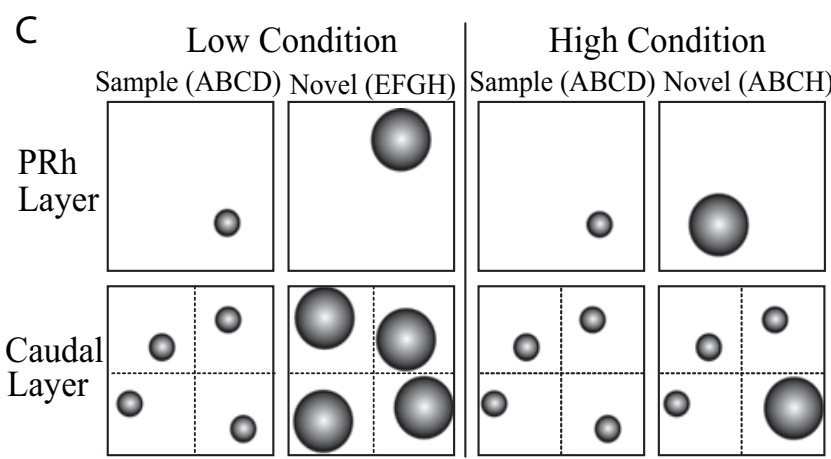

Figure 1. A, Illustration of the connectionist model. The input layer, containing eight nodes, is shown on the far right; the two layers of stimulus representations (PRh and caudal) are shown to the left of the input layer. Stimulus inputs to the network have eight "stimulus dimensions" (attributes); each dimension is represented in the diagram by an individual input node. Stimulus dimensions are paired into four features. Each feature is shown in a different shade of gray and is represented individually on the caudal layer. On the perirhinal cortex layer, the four features are combined into a conjunction, shown in gray, which represents the whole stimulus. $B$, Performance of the model during a zero-delay object-recognition task with two conditions, low and high perceptual similarity. Filled circles represent recognition of the control group and open circles represent recognition scores of the lesion group. $C$, Stimulus representations on the Kohonen grids of the model in the choice phase for the low (left) and high (right) objectrecognition conditions. The PRh layer comprises one Kohonen grid with a single conjunctive representation of an object stimulus; the caudal layer comprises four Kohonen grids with separate representations of the individual features comprising the object stimulus. Small circles indicate sharply tuned ("familiar") representations, and large circles indicate coarsely tuned ("unfamiliar") representations. For discussion, see Materials and Methods, Architecture of the model.

receives stimulus inputs and is characterized by lateral inhibitory feedback between neighboring units. The grids are trained by the successive presentation of a number of stimulus inputs; the weights of each unit are incrementally adapted on each presentation. This results in an automatic mapping of stimulus inputs onto a set of representations that possess the 
same topological order as the stimuli, that is, similar stimuli are represented in neighboring locations on the grid. The self-organization process involves the sharpening of representations of stimuli on which the network is trained. A novel stimulus will elicit a moderate level of activity, broadly distributed across a large number of units in the grid [Cowell et al. (2006), their Fig. 3, top]; as that stimulus is presented repeatedly, the activation pattern it elicits becomes more selective until only a small area of the grid contains highly active units, producing a peak of activation [Cowell et al. (2006), their Fig. 3, bottom]. The development of sharply tuned representations can thus be used as the basis for familiarity judgments: as a stimulus representation becomes sharper, so is it judged to be more familiar (Norman and O'Reilly, 2003). The model may be used to simulate the effects of damage to perirhinal cortex by removing the perirhinal cortex layer so that object recognition performance depends on the caudal layer alone.

\section{Simulation methods}

Stimuli. Object stimuli in this experiment were created by constructing four-featured objects from a pool of 16 possible visual features. Each feature comprises two stimulus dimensions or attributes and each fourfeatured object comprises eight stimulus dimensions. On the caudal layer, each two-dimensional feature is represented as a simple conjunction and a four-featured object is represented as four separate simple conjunctions. On the PRh layer, a four-featured object is represented as a single complex conjunction. Real-world objects may be thought to contain more features than this, but the model is designed to illustrate a principle rather than reproduce the real-world situation strictly veridically.

Four pairs of stimuli were created for the present experiment; each pair comprised a four-featured sample object and a four-featured novel object. Two of the four stimulus pairs were assigned to the "low" condition (that is, the sample and choice object comprising the pair are dissimilar and therefore easy to discriminate) and two pairs were "high" (the sample and novel object are similar and therefore difficult to discriminate). In the low pairs, the sample and novel object shared no features. In the high condition, the sample and novel object shared three of four features. Neither the sample nor the novel stimulus in any pair was replicated in any other pair, but individual features were allowed to appear in more than one object pair.

Simulation procedure. Two groups of 12 networks were tested: the "control" group consisted of intact networks and the "PRh lesion" group consisted of networks in which the PRh layer had been removed to simulate PRh lesions. Each network was tested on two object sets under each condition, low and high, giving four trials per network. Networks were initialized and pretrained before testing on the four trials [for details, see Cowell et al. (2006), their Appendix 1]. On each trial, a network was presented with the sample object and allowed to "encode" the object for 20 cycles; each cycle sharpened incrementally the peak of activation representing the sample object [Cowell et al. (2006), their Appendix 1]. After encoding, each network was presented with both the sample and the novel object in a "choice" phase. No learning occurred in the choice phase; the representations of the two objects were simply assessed to obtain an index of their relative familiarity (the recognition score). At the beginning of every new trial, each network was reset to the state it had assumed at the end of pretraining.

\section{Experiment 2}

In experiment 2 we assessed the performance of PPRh-lesioned and control rats in a zero-delay object-recognition task under two different perceptual conditions. Performance in a zero-delay object-recognition task is regarded as an assay of perceptual (and not mnemonic) function (Eacott et al., 1994; Buffalo et al., 1999, 2000; Holdstock et al., 2000; Levy et al., 2005), as there is little or no memory demand. Because PRh has been implicated in complex visual discrimination performance, and as illustrated in the simulations in experiment 1 , we predicted that PPRhlesioned rats would be impaired in a zero-delay object-recognition task when the novel stimulus was designed explicitly to resemble the sample stimulus (high condition), but would perform similarly to the control group when the novel stimulus did not share features in common with the sample stimulus (low condition). Such a PPRh impairment with a zero delay would suggest that $\mathrm{PRh}$ in the rat is not only important for memory, but might also be necessary for the perceptual processing of complex stimuli.

\section{Subjects}

The subjects were 24 adult male Lister hooded rats (Harlan Olac, Bicester, UK) weighing 270-320 g before surgery and housed in pairs in a room with a $12 \mathrm{~h}$ light/dark cycle (lights on at 7:00 P.M.). All behavioral testing was conducted during the dark phase of the cycle. These rats were tested previously in a short object recognition pilot experiment; no objects from this pilot experiment were reused in the present experiment. During testing, rats were fed $\sim 15 \mathrm{~g}$ of laboratory chow after daily behavioral sessions to maintain weights at $85-90 \%$ of free-feeding body weight. Water was available ad libitum throughout the experiment. All experimentation was conducted in accordance with the United Kingdom Animals (Scientific Procedures) Act (1986).

\section{Surgery}

Rats were divided into two groups: PPRh lesions $(n=11)$ and surgical controls $(n=13)$. Before surgery, all animals were deeply anesthetized by intraperitoneal injection $(60 \mathrm{mg} / \mathrm{kg}$, i.p.) of sodium pentobarbital (Sagatal; Rhône Mérieux, Essex, UK) and placed in a stereotaxic frame (Kopf Instruments, Tujunga, CA) with the incisor bar set at +5.0. The scalp was cut and retracted to expose the skull. Craniotomies were then performed directly above the target region, and the dura was cut to expose the cortex.

For the PPRh lesions, injections of $0.2 \mu \mathrm{l}$ of 0.9 M NMDA (Sigma, Poole, UK) dissolved in phosphate buffer, $\mathrm{pH}$ 7.4, were made through a $1 \mu \mathrm{l}$ Hamilton syringe into five sites in each hemisphere. Each injection was made gradually over a 2 min period, and the needle was left in situ for an additional 4 min before being withdrawn. The stereotaxic coordinates relative to ear-bar zero were as follows: anteroposterior (AP) +3.9, lateral (L) \pm 5.9 , dorsoventral $(\mathrm{DV})+2.0$; $\mathrm{AP}+2.4, \mathrm{~L} \pm 6.1, \mathrm{DV}+1.6$; $\mathrm{AP}$ $+0.6, \mathrm{~L} \pm 6.2, \mathrm{DV}+2.5 ; \mathrm{AP}-0.8, \mathrm{~L} \pm 6.2, \mathrm{DV}+2.7$; and $\mathrm{AP}-0.8, \mathrm{~L}$ $\pm 6.2, \mathrm{DV}+4.3$.

Control animals received sham PPRh surgeries. For sham surgeries, the same initial surgery was performed (including craniotomy and insertion of needle), but no injections were made. At the completion of surgery, the skin was sutured, and an antibiotic powder (Acramide; Dales Pharmaceuticals, Skipton, UK) was applied. Animals were then administered subcutaneously with $5 \mathrm{ml}$ of glucose saline (Aquapharm; Animalcare Limited, York, UK).

\section{Histology}

After behavioral testing, rats were anesthetized by intraperitoneal injection of $2 \mathrm{ml}$ of Euthatal (Rhône Mérieux) and perfused transcardially with $100 \mathrm{ml}$ of PBS, pH 7.4, followed by $250 \mathrm{ml}$ of $4 \%$ paraformaldehyde (PFA), $\mathrm{pH}$ 7.4. The brains were removed, postfixed in $4 \% \mathrm{PFA}$ at $4^{\circ} \mathrm{C}$ for $24 \mathrm{~h}$, and then immersed in $25 \%$ sucrose in PBS until they sank. Coronal sections $(60 \mu \mathrm{m})$ were cut on a freezing microtome through the extent of the lesioned area, and every fifth section was mounted on a gelatincoated glass slide and stained with cresyl violet. Slides were examined under a light microscope to determine the extent of excitotoxin-induced damage.

\section{Spontaneous object recognition}

Apparatus. Spontaneous object recognition was conducted in a Y-shaped apparatus as described previously (Forwood et al., 2005). Briefly, the Y-shaped apparatus had high, homogeneous white walls constructed from Perspex (Lucite International, Southampton, UK) to prevent the rat from looking out into the room and thereby maximizing attention to the stimuli. All walls were $40 \mathrm{~cm}$ high and each arm was $27 \mathrm{~cm}$ in length and $10 \mathrm{~cm}$ wide. The start arm contained a guillotine door $18 \mathrm{~cm}$ from the rear of the arm. This provided a start box area within which the rat could be confined at the start of a given trial. The floor and walls were wiped down with a dry paper towel between trials but otherwise were not cleaned during the experiment. A lamp illuminated the apparatus and a white shelf $50 \mathrm{~cm}$ from the top of the apparatus created a ceiling on which a video camera was mounted to record trials. 
Zero-delay object recognition. To facilitate immediate viewing between test phases ( $0 \mathrm{~s}$ delay), modifications were made to the original Y-apparatus (Fig. 2A). Four metal posts (two per arm, each $33.4 \mathrm{~cm}$ in height) were inserted and positioned $12 \mathrm{~cm}$ apart from each other, which in turn created holders for one sliding door in each arm of the Y-apparatus. The doors (composed of white Perspex) were $33 \mathrm{~cm}$ tall and $10 \mathrm{~cm}$ wide.

Lego objects. The Lego objects were composed entirely from Lego (Lego Group, Billund, Denmark). All Lego objects were between 8.5 and 11.5 $\mathrm{cm}$ tall and 6.6 and $9.2 \mathrm{~cm}$ wide and were affixed to an $8.5 \times 8.5 \mathrm{~cm}$ black Lego sheet. Lego objects were secured to the floor of the apparatus with Blu-Tack (Bostik, Stafford, UK). As far as could be determined, the Lego objects had no natural significance for the rats, and they had never been associated with a reinforcer.

General procedure. All rats were habituated in two consecutive daily sessions in which they were allowed to explore the empty Y-apparatus for $5 \mathrm{~min}$. For these habituation sessions, the rat was placed in the start box, and the guillotine door was opened to allow the rat to explore the main area of the apparatus. The guillotine door was lowered when the rat exited the start box to prevent re-entry into this area of the apparatus. The experimenter did not begin timing the trial until the rat exited the start box. Testing began $24 \mathrm{~h}$ after the second habituation session. Rats were given a series of test trials (one per day) with a minimum interval of $24 \mathrm{~h}$ between trials. A different object pair was used for each trial for a given animal, and the order of exposure to object pairs as well as the designated sample and novel objects for each pair were counterbalanced within and across groups. The time spent exploring objects was assessed from video recordings of the sample and choice phases. Data were collected by scoring exploratory bouts using a personal computer running a program written in Visual Basic 6.0 (Microsoft, Redmond, WA).

Object recognition test. All object sets used in a given trial were placed in the apparatus before the rat was placed in the start box. The rat was then placed in the start box with the guillotine door lowered. The guillotine door was then raised to allow the rat into the exploration area of the apparatus. When the rat exited the start box, the guillotine door was lowered to prevent re-entry, and the test phases began. The time spent exploring the two objects in a testing phase was scored by an experimenter viewing the rat on a video screen. The cumulative duration of exploratory bouts, the beginning and end of which were indicated by pressing a given key on the computer keyboard, was calculated by the computer program. Exploration of an object was defined as directing the nose to the object at a distance of $<2 \mathrm{~cm}$ and/or touching it with the nose.

The sample phase ended when the rat had explored the identical objects for $25 \mathrm{~s}$ or when $5 \mathrm{~min}$ had passed. At the end of the sample phase, the identical objects were removed and the door between the sample and choice zones was immediately opened, thereby presenting the choice objects to the rat. The choice phase contained an identical copy of the sample (familiar) object in one arm and a novel object in the other. The arm in which the novel object was placed was counterbalanced between rats and across trials. The time spent exploring the novel and familiar objects was recorded for $3 \mathrm{~min}$ of the choice phase, but attention was focused on the first minute, during which object discrimination is typically greatest (Dix and Aggleton, 1999). We calculated a discrimination ratio, the proportion of total exploration time spent exploring the novel object (i.e., the difference in time spent exploring the novel and familiar objects divided by the total time spent exploring the objects), for the first minute of the choice phase on each object recognition trial. This measure takes into account individual differences in the total amount of exploration time.

Rats were tested in two conditions, low and high; the presentation order of the two conditions was counterbalanced between rats and across trials.

Low condition. In the low condition, two identical Lego objects (A1 and A2) were presented in the sample phase. During the choice phase, the apparatus contained an identical copy of the sample (familiar) object (A3) in one arm and a new Lego object (B) in the other (see Experiment 3, Stimuli) (Fig. 2C). The novel Lego object was constructed to have minimal perceptual similarity to the familiar object. The novel and familiar Lego objects were constructed to share minimal features in com- mon with one another; minimal perceptual similarity was achieved by minimizing the number of colors and features shared between the two Lego objects.

High condition. The procedure in the high condition was identical to that in the low condition, except that the novel Lego object was explicitly constructed to share features in common with the familiar object (see Experiment 3, Stimuli) (Fig. 2C).

\section{Data analysis}

Group means of three measures taken from object recognition testing [duration of sample phase (i.e., the time taken to accumulate criterion levels of exploration in the sample phase), total exploration time in the choice phase, and the discrimination ratio] were analyzed. Means from each of the three measures were submitted to a two-way ANOVA where the first factor was the between-subjects factor of lesion group and the second factor was the within-subjects factor of condition. Significant interaction effects were further analyzed with independent sample $t$ tests. All tests of significance were performed at $\alpha=0.05$.

\section{Experiment 3}

In experiment 2, impairments after lesions to PPRh were revealed in the zero-delay object-recognition task, and these deficits emerged only when the stimuli to be discriminated in the choice phase were perceptually similar to one another, suggesting a perceptual impairment. However, it might be argued that this was still a mnemonic impairment, albeit one that is revealed only under conditions of high perceptual load, because the stimuli to be discriminated were not presented simultaneously. Therefore we tested the effects of PPRh and PRh lesions on a simultaneous oddity discrimination test; in addition, we added sets of objects to provide a broader continuum of perceptual similarity (the results are presented in experiment 4). Before experiment 4 , however, we sought to determine different levels of perceptual difficulty using a simple visual discrimination task to ensure that stimuli that shared many features in common were more difficult for rats to discriminate than the stimuli that shared fewer features in common. Therefore, we pretested these sets of stimuli with naive rats in a two-choice discrimination procedure to provide a measure of the subjective perceptual similarity of the object in each pair. Naive rats were tested in four perceptual conditions: low, medium, medium-high, and high. The low and high object sets were the same as those used in experiment 2 . The objects within each pair in the two new sets were designed to have intermediate perceptual similarities.

\section{Subjects}

Sixteen experimentally naive Lister hooded rats (weighing 270-320 g) were used for experiment 3 . The animals were housed and fed in the same manner as the rats used in experiment 2. Four rats were tested per perceptual condition.

\section{Visual discrimination}

Apparatus. The same Y-apparatus was used as in experiment 2. However, modifications were made to the apparatus to accommodate discrimination testing. In each of the arms, a Foamalux insert $(40 \mathrm{~cm}$ tall and $9.9 \mathrm{~cm}$ wide) was added where there was previously a door between the sample and choice zones. The insert blocked the areas of the apparatus that were not used during testing. Small food wells $(4.5 \mathrm{~cm}$ in diameter) were placed $12 \mathrm{~cm}$ from the start of the Y-apparatus in each arm and were located against the left side of both of the arms. Stimuli were placed directly beside the food wells.

Stimuli. For pretraining two wooden blocks (one white and one black) were used ( $9.5 \mathrm{~cm}$ tall and $9.5 \mathrm{~cm}$ wide). Wood was chosen because none of the stimuli used in the discrimination experiment proper were made of wood. For the discrimination experiment proper, four different stimulus conditions were used: low, medium, medium-high, and high. The stimuli used for the low and high conditions were the same stimuli used during the choice phase of experiment 2. For the new conditions, medium and medium-high, new Lego objects were constructed with varying levels of difficulty (Fig. 2C). Lego stimuli were constructed in a similar manner to experiment 2. The stimuli in the medium condition were constructed to share a few features in common whereas the stimuli in the 

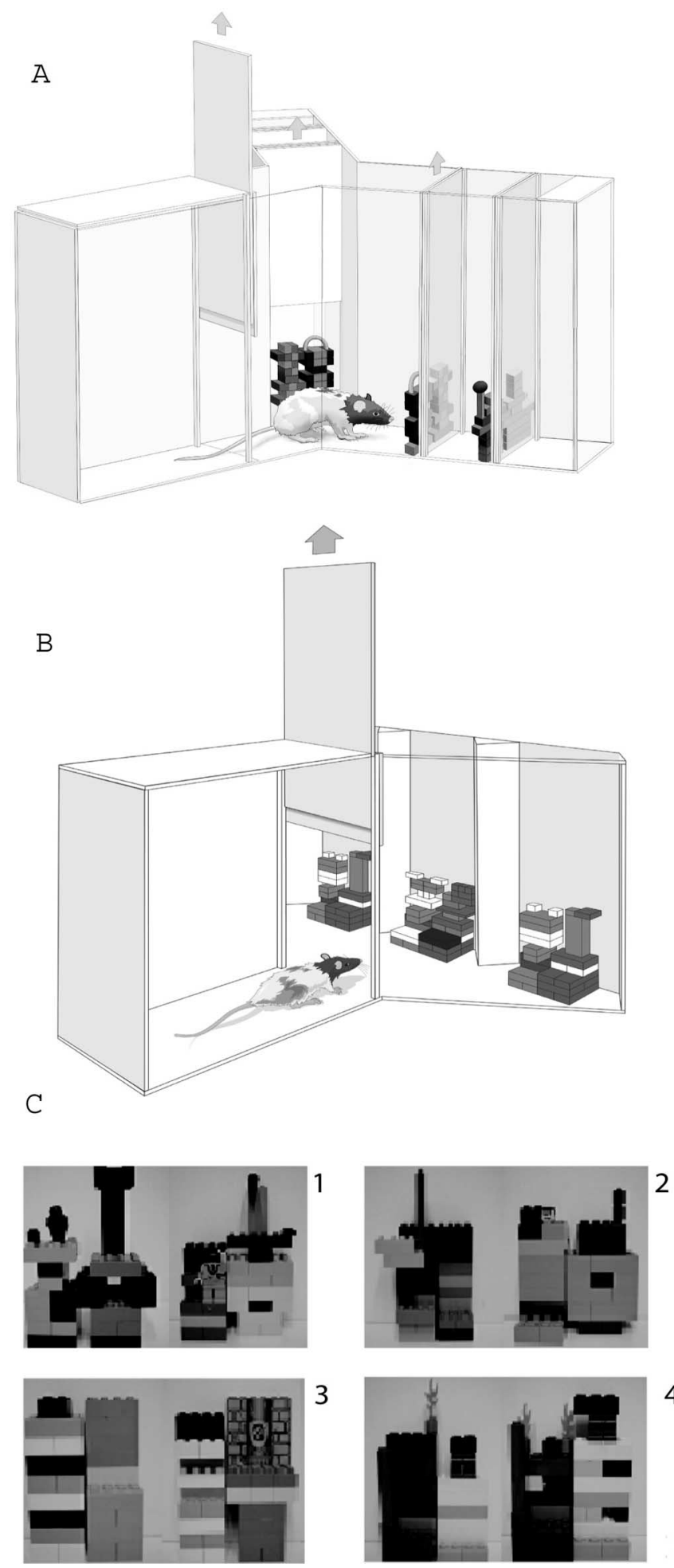

Figure 2. A, lllustration of the apparatus and representative stimuli used in the zero-delay object-recognition task in experiment 2. The figure illustrates examples of stimuli that could appear in the low condition during a given trial. The nearest wall appears transparent for illustrative purposes and the guillotine door is shown raised. The sample objects are closest to the rat; the choice objects comprise the next set of objects behind the sample objects. The door behind the choice objects remained closed during all testing. All stimuli (sample and choice objects) were placed in the apparatus before testing began. At the beginning of a trial, the rat was released from the start box when the experimenter raised the guillotine door. In the sample phase, the rat was exposed to identical versions of the same object. At the end of the sample phase, the objects were removed and the door between the sample and choice objects was immediately raised. In the choice phase, the rat was exposed to a third, identical copy of the sample at the end of the one exploration arm and a novel Lego object at the end of the other medium-high condition stimuli were constructed to share more features in common than medium, but fewer than the high condition.

Habituation. All rats were given four habituation sessions on separate consecutive days, $24 \mathrm{~h}$ apart, before their first testing day. For habituation sessions one and two, the rat was placed in the start box, the guillotine door opened, and the rat was free to explore the apparatus for $5 \mathrm{~min}$. Nestlé (York, UK) Cheerios were scattered throughout the arms of the apparatus. In habituation sessions three and four, food was placed only in the food wells and the rat was left in the apparatus for $5 \mathrm{~min}$; $24 \mathrm{~h}$ later, testing began.

Pretraining. Pretraining sessions consisted of 25 trials each. Half of the rats were rewarded for approaching the black wooden block and half were rewarded for approaching the white wooden block. The side on which the reward stimulus was presented on a given trial was determined pseudorandomly. Each rat was placed in the start box at the beginning of a trial; the guillotine door was raised and then shut after the rat entered the exploration area of the apparatus. A response was scored as correct if the rat approached (within $0.5 \mathrm{~cm}$ of the correct stimulus) and/or touched the object with its nose. If the rat chose correctly, one half of a Cheerio was placed in the food well directly in front of the stimulus. The experimenter waited for the rat to finish eating, then placed the rat back in the start box. The guillotine door was then raised to continue to the next trial. If a rat performed incorrectly, the rat was placed in the start box for $15 \mathrm{~s}$, and then the same trial was repeated until the correct choice was made. However, only the first choice on a given trial was scored, not the correction trials. The animals had to reach a criterion of $75 \%$ correct (19 of 25) on 2 consecutive days before the discrimination experiment proper began. Discrimination testing did not occur until all rats reached criterion. Because some rats reached criterion before day 11 (after a rat reached criterion, pretraining ended), all rats were run on the pretraining discrimination $1 \mathrm{~d}$ before the visual discrimination experiment, to ensure that they could still perform at criterion (all rats attained 19 of 25 or better).

Visual discrimination. The visual discrimination task consisted of nine sessions of 25 consecutive trials daily, plus correction trials. Four rats were assigned to each stimulus condition (low, medium, medium-high, and high). The side on which the reward stimulus was presented on a given trial was determined pseudorandomly. The experimental testing procedure was identical to the pretraining testing phase.

\section{Data analysis}

Data were analyzed in blocks of three for a total of nine sessions. The average number correct (of 25) was calculated for each group and was analyzed using a univariate analysis of the four means. Significant effects of group were further analyzed with Tukey's honest significant difference (HSD) post hoc tests. All tests of significance were performed at $\alpha=0.05$.

\section{Experiment 4}

Experiment 4 was designed to replicate and extend the findings of experiment 2. Although recognition was tested using a zero-delay condition in experiment 2 , it might be argued that there was still a mnemonic component because, although there was no delay, the rat had to remember the familiar object from the sample phase during the time of the discrimination (in the choice phase) to discriminate the novel stimulus. Therefore, in experiment 4, animals were tested in a novel behavioral paradigm, the simultaneous oddity discrimination task (S. E. Forwood, S. J. Bartko, L. M. Saksida, and T. J. Bussey, unpublished observations), in which all objects were presented simultaneously. Rats were presented

$\leftarrow$

arm. $\boldsymbol{B}$, The spontaneous oddity apparatus used for experiment 4 . All stimuli (two identical and one odd object) were placed in the apparatus before testing began. At the beginning of a trial, the rat was released from the start box when the experimenter raised the guillotine door. Exploration of the two identical objects and the one odd object was recorded by the experimenter. The odd object could appear in any of the three locations; here, it is shown in the center location. C, Examples of stimuli from experiments 2-4; clockwise from top left: low (1), medium (2), medium-high (3), and high (4). For experiment 2, only low and high stimuli were used. All four stimulus conditions were used in experiment 3 and 4. 
with two copies of one object and one copy of another object; that is, three objects were presented, an odd object and two identical objects. We predicted that the rat would divide its exploration between the two identical objects, resulting in an overall "preference" for the odd object. The stimulus pairs identified in experiment 3 as having four different levels of perceptual difficulty served as stimuli for the oddity discrimination task in experiment 4 . Two lesion groups, PPRh and PRh, were tested in addition to a control group. The PRh-only lesion group was added to localize the lesion to PRh and to reduce and possibly eliminate any effects attributable to area TE damage.

\section{Subjects}

Forty-two experimentally naive Lister hooded rats (weighing 270-320 g) were used for experiment 4 . The animals were housed and fed in the same manner as the rats used in experiments 2 and 3.

\section{Surgery}

Rats were divided into three groups: PPRh lesions (PPRh) ( $n=14), \mathrm{PRh}$ lesions (PRh) $(n=14)$, and surgical controls (control) $(n=14)$. Surgeries for the PPRh and control groups were identical to those in experiment 2 except no needle was inserted into the lesion site for the control group (only a craniotomy was performed).

For the PRh lesions, injections of $0.2 \mu \mathrm{l}$ of $0.9 \mathrm{M} \mathrm{NMDA}$ (Sigma) dissolved in phosphate buffer, $\mathrm{pH}$ 7.4, were made through a $1 \mu \mathrm{l} \mathrm{Ham}$ ilton syringe into three sites in each hemisphere. Each injection was made gradually over a 2 min period, and the needle was left in situ for an additional 4 min before being withdrawn. The stereotaxic coordinates relative to ear-bar zero were as follows: $\mathrm{AP}+3.9, \mathrm{~L} \pm 5.9, \mathrm{DV}+2.0$; $\mathrm{AP}$ $+2.4, \mathrm{~L} \pm 6.1, \mathrm{DV}+1.6$; and $\mathrm{AP}+0.6, \mathrm{~L} \pm 6.2, \mathrm{DV}+2.5$.

\section{Histology}

The same histological protocol was used as described previously for experiment 2.

\section{Oddity task}

Apparatus. The oddity apparatus incorporated the same considerations used to design the Y-apparatus (Forwood et al., 2005) (Fig. 2 B). The exploration area was triangular in shape. The oddity apparatus had high, homogenous white walls constructed from Perspex (Lucite International) to prevent the rat from looking out into the room. All walls were $40 \mathrm{~cm}$ high and the start box contained a guillotine door $25 \mathrm{~cm}$ from the rear of the box, providing a start box area within which the rat could be confined at the start of a trial. The back wall (bottom part of the triangle) was positioned $19.5 \mathrm{~cm}$ from the guillotine door. The back wall was $35 \mathrm{~cm}$ long with four $3 \mathrm{~cm}$ wide dividers positioned between the three $10 \mathrm{~cm}$ spaces (where the objects were placed) along the back wall.

Lego objects. Four different perceptual conditions were tested in experiment 4 . These were the low, medium, medium-high, and high condition stimulus pairs identified in experiment 3 .

General procedure. All rats were habituated in two consecutive daily sessions in which they were allowed to explore the empty oddity apparatus for $5 \mathrm{~min}$. For these habituation sessions, the rats were placed in the start box and the guillotine door was opened to allow the rat to explore the main area of the apparatus. The guillotine door was lowered when the rat exited the start box to prevent re-entry into this area of the apparatus. The experimenter did not begin timing the trial until the rat exited the start box. Testing began $24 \mathrm{~h}$ after the second habituation session. Rats were given a series of test trials (one per day) with a minimum interval of $24 \mathrm{~h}$ between trials. A different object trio was used for each trial for a given animal, and the order of exposure to object pairs, the designated repeated objects and odd object for each pair, and the odd object location were counterbalanced within and across groups. The time spent exploring objects was assessed from video recordings of the trials. Data were collected by scoring exploratory bouts using a personal computer running a program written in Visual Basic 6.0 (Microsoft).

Oddity test. All object sets used in a given trial were placed in the apparatus before the rat was placed in the start box. The rat was then placed in the start box with the guillotine door lowered. The guillotine door was then raised to allow the rat into the exploration area of the apparatus. When the rat exited the start box, the guillotine door was lowered to prevent re-entry, and testing began. The time spent exploring the three objects during a testing phase was scored by an experimenter viewing the rat on a video screen. The cumulative duration of exploratory bouts, the beginning and end of which were indicated by pressing a given key on the computer keyboard, was calculated by the computer program. Exploration of an object was defined as directing the nose to the object at a distance of $<2 \mathrm{~cm}$ and/or touching it with the nose.

Exploration of two identical and one odd object was recorded for 5 min. We calculated an oddity preference percentage score, the exploration of the odd object divided by the total exploration of the odd and identical objects. Using this score, an oddity preference score of $33.0 \%$ would indicate chance performance (the rat explored all objects equally). An oddity preference score of $100.0 \%$ would indicate total preference for the odd object. Although an oddity preference score of $100.0 \%$ indicates maximum preference for the odd object, this score is not a realistic score because a score this high can only be revealed if the rat only explores the odd object. In reality, the rat must explore all objects before preference will occur for the odd object. Therefore, an oddity preference score significantly $>33.3 \%$ (or chance performance) would represent a meaningful score on this task.

Rats were tested in four conditions: low, medium, medium-high, and high. The presentation order of the four conditions was counterbalanced between rats and across trials.

\section{Data analysis}

Total object exploration during the oddity task (total exploration of the odd and identical objects) was analyzed because a difference in exploration could affect oddity preference. Preference for the odd object (exploration of the odd object divided by total exploration) was also analyzed. Means from each of these measures were submitted to a two-way ANOVA where the first factor was the between-subjects factor of lesion group and the second factor was the within-subjects factor of condition. Significant interaction effects were further analyzed with independent samples $t$ tests. All tests of significance were performed at $\alpha=0.05$.

\section{Results}

\section{Experiment 1}

As shown in Figure $1 B$, networks in both the control group and lesion group performed well on object recognition in the low perceptual difficulty condition. Both groups performed more poorly in the high perceptual difficulty condition than in the low condition. However, a clear group difference, not seen in the low condition, was revealed between the groups in the high condition: the control group was still able to discriminate the novel and familiar stimuli whereas networks in the lesion group were unable to make the discrimination. Thus, the model predicts that introducing a high level of perceptual similarity between the sample and choice objects will cause impairments in the object recognition performance of subjects with PRh lesions relative to the performance of control subjects. This prediction of the model arises because, whereas the intact networks can represent the conjunction of features of a stimulus as well as the individual features, the lesioned networks can represent only the individual stimulus features (Fig. 1C). In the low condition, two dissimilar objects are presented to networks in the choice phase, one of which is familiar and one of which is novel, and both layers of the model are able to discriminate the stimuli on the basis of familiarity. On the caudal layer, where individual features of stimuli are represented separately, all four features are sharply tuned for the familiar stimulus whereas all four are coarsely tuned for the novel stimulus. On the PRh layer, the single conjunctive representation of the familiar stimulus is sharply tuned and can be discriminated from the coarsely tuned conjunctive representation of the novel stimulus. However, in the high condition the novel and familiar objects share three features. This means that on the caudal layer of the model, three of the four features pos- 
sessed by the novel stimulus appear familiar because their representations have been tuned through encoding of the sample stimulus; the stimulus representations are therefore much less discriminable on the basis of familiarity. In contrast, on the PRh layer, the two conjunctive representations of the familiar and novel stimuli are distinct so that only the familiar representation is sharply tuned and, therefore, the novel and familiar objects are readily discriminable. One can see from Figure $1 C$ that removing the rostral (PRh) layer, leaving only the caudal layer to solve the discrimination, will result in a behavioral impairment.

\section{Experiment 2 \\ Histology}

Throughout this study, histological assessment was made with reference to the anatomical designations of Burwell (2001). In the PPRh group, extensive cellular loss was revealed through the perirhinal and postrhinal cortices (Fig. 3). The lesion was observed in the rostral border of PRh and continued caudally throughout perirhinal and postrhinal cortices. The lesion also extended ventrally to include the lateral entorhinal cortex and the piriform cortex in all PPRh animals. There was some unilateral sparing of the most rostral PRh in one animal. Minor unilateral damage to area CA1 in the ventral hippocampus was observed in one animal.

Minor, incidental TE damage was observed in all PPRh animals (bilateral in six PPRh rats and unilateral in five PPRh rats). However, comparison of the mean discrimination ratios between rats with unilateral TE damage or rats with bilateral TE damage showed no significant group effect $\left(F_{(1,9)}=2.02\right)$ and no interaction with condition $(F<1)$.

Histological analysis revealed no cellular loss in the perirhinal and postrhinal cortices of the control group. However, unilateral cortical damage was observed in two control rats, visible in the frontal and parietal cortices from 0.49 to $3.60 \mathrm{~mm}$ posterior to bregma. The damage is possibly a result of the craniotomies or from inserting the needle.

\section{Spontaneous object recognition}

Duration of the sample phase. In the present study, all animals explored the sample for $25 \mathrm{~s}$ in under $5 \mathrm{~min}$ on all trials. The total time required to complete $25 \mathrm{~s}$ of exploration in the sample phase was analyzed significant group differences at this stage of a trial might lead to differences in subsequent recognition performance. This analysis revealed no significant difference between the groups $\left(F_{(1,22)}=1.7\right)$ and no significant effect of condition $\left(F_{(1,22)}=1.92\right)$. The interaction of group by condition was also not significant $(F<1)$. The mean sample phase duration $( \pm$ SEM) for groups in each condition was as follows: low condition, PPRh, $103.50 \pm 11.0 \mathrm{~s}$, control, $92.50 \pm 5.60 \mathrm{~s}$; high condition, PPRh, $95.10 \pm 8.40 \mathrm{~s}$, control, $85.30 \pm 3.90 \mathrm{~s}$.

Object exploration during choice phase. Analysis of the total mean object exploration during the choice phase revealed no

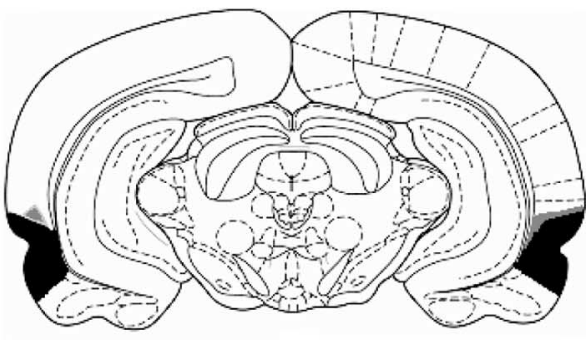

Bregma - $6.04 \mathrm{~mm}$

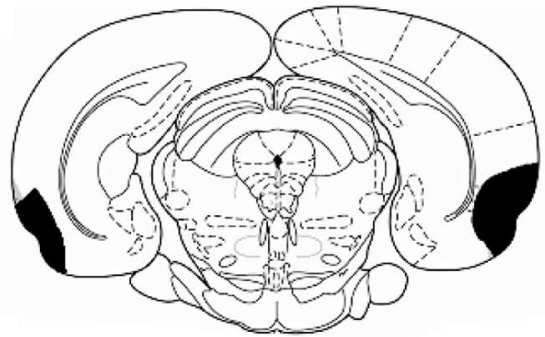

Bregma - $7.04 \mathrm{~mm}$

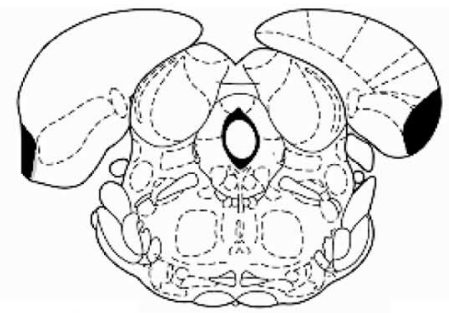

Bregma $-8.72 \mathrm{~mm}$

Figure 3. Coronal sections illustrating the extent of the largest (gray) and smallest (black) lesions of the perirhinal and postrhinal cortex in experiment 2, from 3.14 to $8.72 \mathrm{~mm}$ posterior to bregma (Paxinos and Watson, 1997).

significant group effect $(F<1)$. The PPRh and control groups combined explored the novel and familiar choice objects more in the low condition than in the high condition $\left(F_{(1,22)}=8.03 \mathrm{~s} ; p=\right.$ 0.01 ) (low condition: PPRh, $9.46 \pm 0.71 \mathrm{~s}$, control, $10.08 \pm 0.86 \mathrm{~s}$; high condition: PPRh, $8.11 \pm 0.77 \mathrm{~s}$, control, $7.97 \pm 0.42 \mathrm{~s}$ ). Importantly, however, the interaction of group by condition was not significant $(F<1)$.

Recognition during the choice phase. The PPRh group performed significantly worse than the control group in the high condition but not in the low condition (Fig. 4). A two-way ANOVA with repeated measures conducted on the discrimination ratio revealed main effects of group $\left(F_{(1,22)}=26.17 ; p<\right.$ $0.0001)$, condition $\left(F_{(1,22)}=35.95 ; p<0.0001\right)$, and a significant group by condition interaction $\left(F_{(1,22)}=15.88 ; p=0.001\right)$. Post hoc comparisons with independent samples $t$ tests revealed no significant effect in the low condition $\left(t_{(22)}=1.52\right)$ but a highly significant effect in the high condition $\left(t_{(22)}=0.80 ; p<0.0001\right)$. Moreover, PPRh performance in the high condition was significantly lower than chance (a discrimination ration of zero) $\left(t_{(10)}=\right.$ $-3.40 ; p<0.05$ ). Thus, PPRh-lesioned rats were unable to recognize the novel stimulus in the choice phase and were impaired relative to controls, only when the stimuli were perceptually similar. When the stimuli were perceptually dissimilar, the PPRh and the control groups did not differ: both groups could discriminate the novel from the familiar stimulus in the choice phase.

A relatively minor yet intriguing aspect of the data in the present study is the finding that rats with PPRh lesions performed significantly below chance in the high perceptual similarity condition. Although this result is not without precedent (Mumby et 


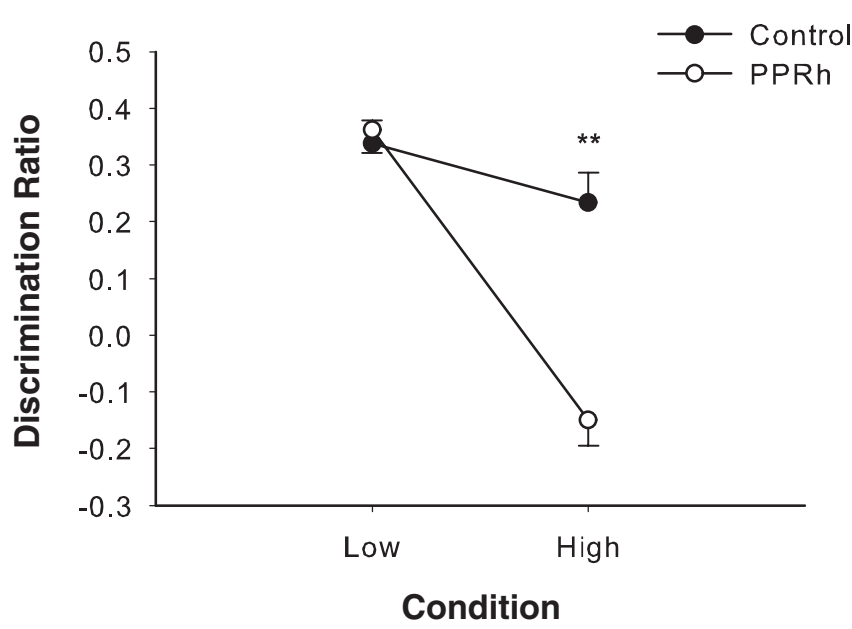

Figure 4. Performance on zero-delay spontaneous object recognition (experiment 2). Data are presented as average discrimination ratio \pm SEM. ${ }^{* *} p<0.0001$.

al., 2002), the reason for it is not immediately obvious. An observation that may be relevant, however, is our finding from pilot studies that during the choice phase, once rats have explored the novel object for a certain amount of time, the novel object becomes more familiar than the previously more familiar (sample) object; the rat then switches its exploration toward the sample object, and the discrimination ratio becomes, at that point, negative. Eventually, the sample object again becomes familiar, and so the rat switches exploration back to the (originally) novel object. We found that rats may cycle through this pattern indefinitely. Thus, it is conceivable that a lesion, which alters the degree to which the novel and familiar objects are perceived as such, might, under certain conditions, lead to the lesioned rats' cycles being "out of phase" with that of the control rats and, depending on the time point at which the discrimination score is calculated, result in a negative discrimination score. As might be expected, negative discrimination scores occur rarely after PRh lesions, and so an account such as this, although obviously speculative, seems to us more likely to be correct than the alternative suggestion that PRh lesions reverse an animal's natural preference for novel objects to a preference for more familiar ones (Mumby, 2001). Moreover, a general shift of preference to more familiar objects would be expected to be revealed at short delays as well as long delays, which it is not.

\section{Experiment 3}

A univariate analysis of the four means on the third block of the nine sessions revealed a highly significant effect of group $\left(F_{(1,15)}=27.03 ; p<0.0001\right.$ ) (Fig. 5). Analysis using a Tukey's HSD post hoc test revealed that performance of the naive rats in the high condition was significantly lower than performance in the low condition $(p<0.0001)$. Discrimination performance in the medium condition was higher than performance in the medium-high condition $(p<0.05)$. Furthermore, discrimination performance in the medium-high condition was significantly lower than performance in the low condition $(p<0.0001)$ and discrimination performance in the medium condition was significantly higher than performance of rats in the high condition $(p<0.0001)$. Thus, the highest discrimination accuracy in the visual discrimination performance was in the lower perceptual difficulty conditions (low and medium) and the poorest discrimination performance was in the higher perceptual difficulty conditions (medium-high and high). The discrimination perfor-

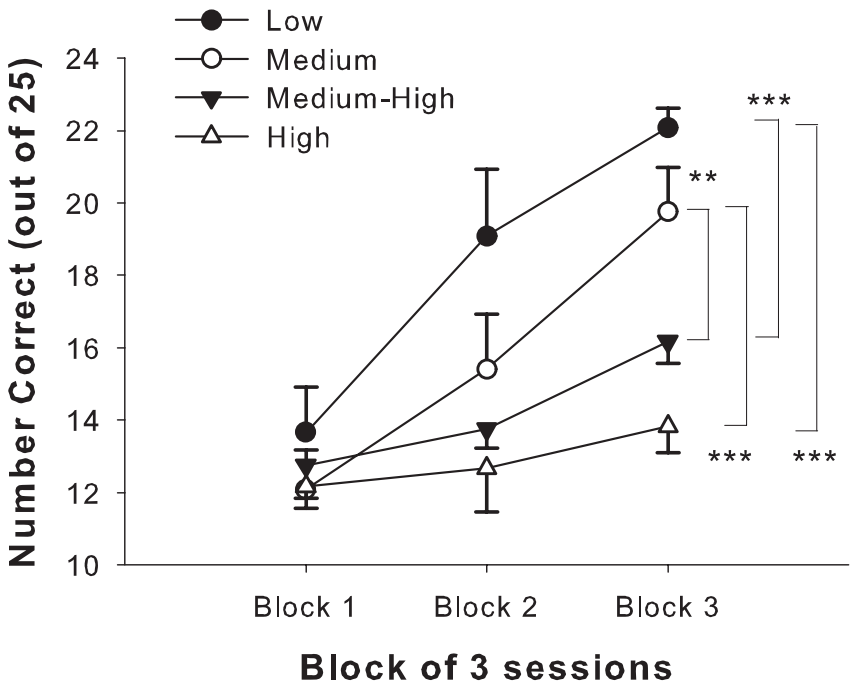

Figure 5. Visual discrimination performance during the four different conditions (low, medium, medium-high, and high) by naive rats in experiment 3. Data are presented as average number correct for nine sessions (presented in blocks of 3 sessions) \pm SEM. ${ }^{* *} p<0.05$; ${ }^{* * *} p<0.0001$.

mance of naive rats in this task confirmed that the stimuli, which shared more perceptual features in common, were more difficult for rats to discriminate than stimuli sharing fewer features in common. These four perceptual conditions were tested in an oddity discrimination task in experiment 4.

\section{Experiment 4}

Histology

Histological analysis revealed no cellular loss in either perirhinal or postrhinal cortices of the control group. However, unilateral $(n=2)$ and bilateral $(n=2)$ cortical damage was observed in four control rats, visible in the frontal and parietal cortices from 0.49 to $3.14 \mathrm{~mm}$ posterior to bregma.

In the PPRh group, extensive cellular loss was revealed throughout the perirhinal and postrhinal cortices. The lesion was observed at the rostral border of PRh and continued caudally throughout the perirhinal and postrhinal cortices. There was some unilateral sparing of the most rostral PRh in two animals. Cortical damage was observed in three (unilateral, $n=2$; bilateral, $n=1$ ) animals, similar to the damage in the control group, possibly caused during the craniotomy. Minor, incidental TE damage was observed in seven animals. Four PPRh-lesioned rats did not sustain substantial damage bilaterally to the perirhinal and postrhinal cortices and were therefore omitted from data analyses.

In the PRh group (Fig. 6), cellular loss was revealed throughout PRh. No TE damage was observed in any of the PRh-lesioned animals (Fig. 7). The lesion was limited to PRh and extended approximately from 3.14 to $7.04 \mathrm{~mm}$ posterior to bregma in most animals. There was some unilateral sparing of the most rostral $\mathrm{PRh}$ in three animals. Four PRh-lesioned rats did not incur substantial damage to $\mathrm{PRh}$ and were omitted from behavioral analyses.

Minor, incidental entorhinal damage was observed in all PPRh animals (bilateral in three PPRh rats and unilateral in seven $\mathrm{PPRh}$ rats). However, comparison of the mean odd object percentage scores between rats with unilateral entorhinal damage or rats with bilateral entorhinal damage showed no significant group effect $\left(F_{(1,8)}=1.97\right)$ and no interaction with condition 
$(F<1)$. Six rats from the PRh group (bilateral in two rats, unilateral in four rats, and no damage in four rats) incurred entorhinal damage from the PRh lesions. However, comparison of the mean odd object percentage scores between rats with unilateral entorhinal damage, rats with bilateral entorhinal damage, and rats with no damage to entorhinal cortex showed no significant group effect $(F<1)$ and no interaction with condition $(F<1)$. Furthermore, analysis using a Tukey's HSD post hoc test revealed that the oddity discrimination performance of $\mathrm{PRh}$ rats with unilateral, bilateral, and animals without damage to entorhinal cortex was not significantly different from one another $(p>$ 0.05 in all cases).

\section{Oddity}

Object exploration during the oddity task. Total exploration of the odd and the identical objects during the oddity task was not affected by lesion. ANOVA revealed no significant interaction of group by condition $\left(F_{(6,93)}=1.53\right)$, and no main effects of group $(F<1)$ or condition $\left(F_{(3,93)}=\right.$ 1.92). The mean exploration during the oddity discrimination task $( \pm$ SEM) for each group in each condition was as follows: low, control, $52.08 \pm 1.84 \mathrm{~s}$, PRh, $45.74 \pm 2.92 \mathrm{~s}$, and PPRh, $48.93 \pm 4.93 \mathrm{~s}$; medium, control, $47.74 \pm 2.82 \mathrm{~s}, \mathrm{PRh}$, $43.64 \pm 2.64 \mathrm{~s}$, and PPRh, $48.21 \pm 4.71 \mathrm{~s}$; medium-high, control, $43.52 \pm 2.20 \mathrm{~s}$, PRh, $42.74 \pm 2.74 \mathrm{~s}$, and PPRh, $48.70 \pm$ $3.92 \mathrm{~s}$; and high, control, $41.17 \pm 2.95 \mathrm{~s}$, $\mathrm{PRh}, 45.67 \pm 3.71 \mathrm{~s}$, and PPRh, $47.91 \pm$ $3.61 \mathrm{~s}$.

Preference for the odd object. The odd object preference score for each group was analyzed for the third minute of exploration during the oddity task, because this was the first time point at which the control group showed a significant preference for the odd object in all conditions. Analysis of the percent preference for the odd object at 3 min revealed a significant main effect of condition $\left(F_{(3,93)}=18.47 ; p<\right.$ $0.0001)$ and a significant group effect $\left(F_{(1,31)}=5.29 ; p=0.003\right)$. The interaction of group by condition was also significant $\left(F_{(6,93)}=1.67 ; p<0.05\right)$ (Fig. 8). Post hoc analysis of the group effect with Tukey's HSD revealed that both the PRh and PPRh group were significantly impaired relative to the control group (PRh, $p<0.05$; PPRh, $p<0.05)$. The two lesion groups, PPRh and PRh, did not differ from one another $(p>0.05)$.

Post hoc comparisons of condition with independent samples $t$ tests revealed no significant difference of the groups in the
Bregina - 3.14

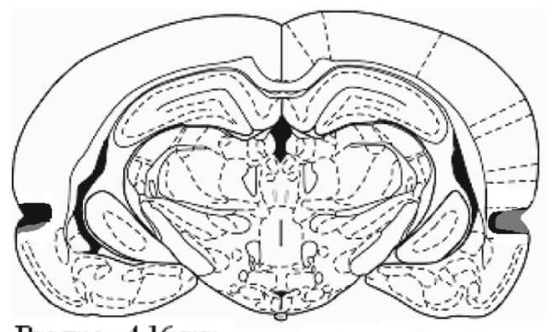

Bregma - 4.16 mm

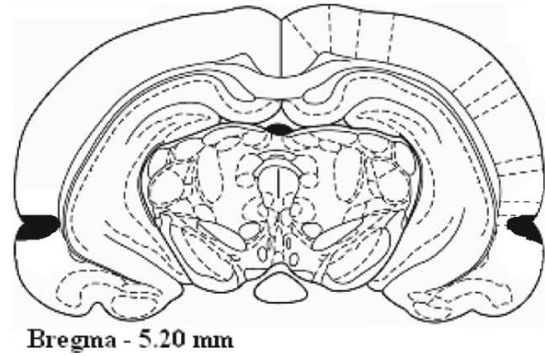

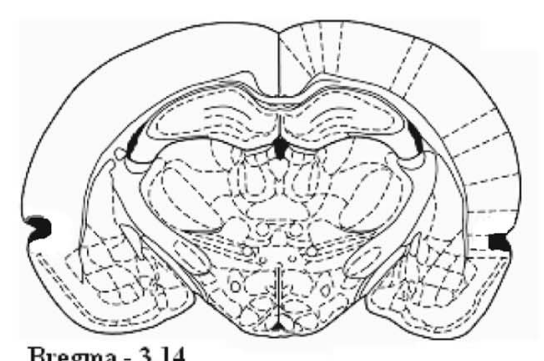

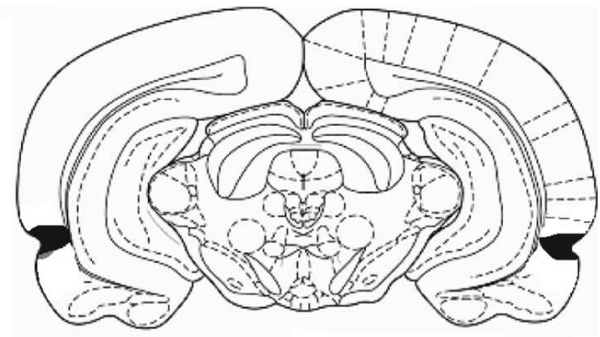

Bregna - $6.04 \mathrm{~mm}$

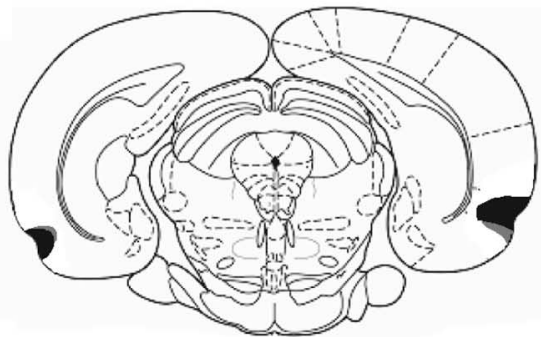

Bregma - $7.04 \mathrm{~mm}$

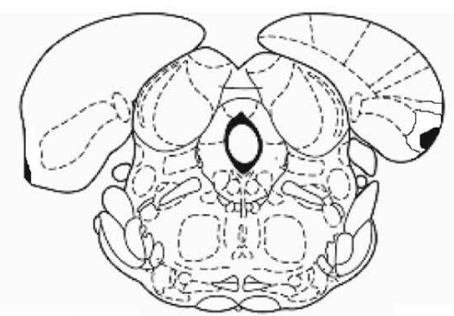

Bregma - $8.72 \mathrm{~mm}$
Figure 6. Coronal sections illustrating the extent of the largest (gray) and the smallest (black) lesions of the perirhinal cortex in experiment 4, from 3.14 to $8.72 \mathrm{~mm}$ posterior to bregma (Paxinos and Watson, 1997).
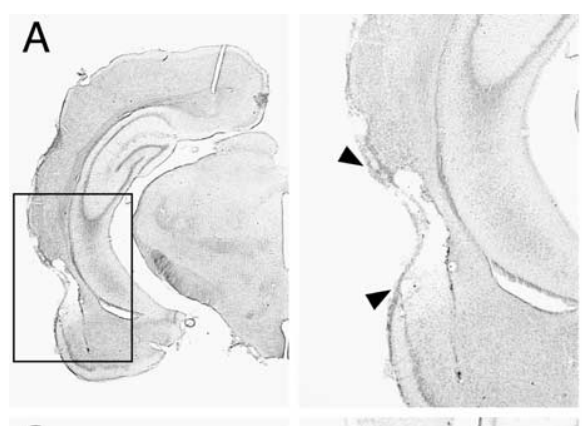

B
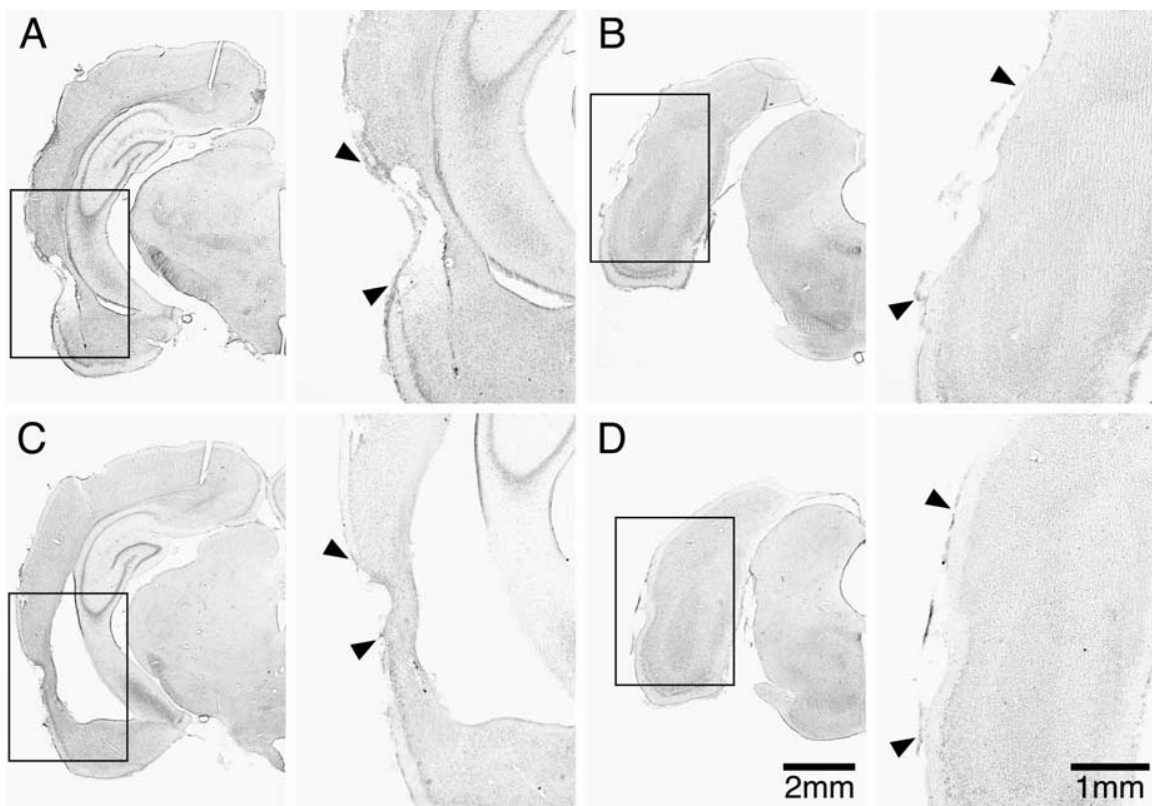

D
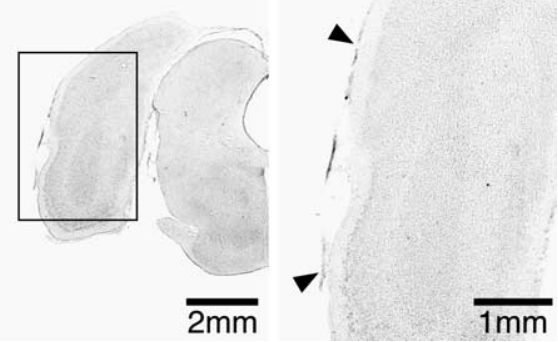

Figure 7. $\boldsymbol{A}-\boldsymbol{D}$, Photomicrographs illustrating lesions from the PPRh $(\boldsymbol{A}, \boldsymbol{B})$ and $\operatorname{PRh}(\boldsymbol{C}, \boldsymbol{D})$ groups from experiment 4. $\boldsymbol{A}$, Typical PRh damage from the PPRh lesion, shown here at $\sim 4.8 \mathrm{~mm}$ posterior to bregma (Paxinos and Watson, 1997). $\boldsymbol{B}$, Postrhinal cortex damage from the PPRh lesion at $\sim 8.0 \mathrm{~mm}$ posterior to bregma. $C$, Perirhinal cortex damage from the PRh lesion, shown here at $\sim 4.8 \mathrm{~mm}$ posterior to bregma. $D$, Intact postrhinal cortex after $P R h$ lesion, shown here at $\sim 8.12 \mathrm{~mm}$ posterior to bregma. Note that there is no damage to postrhinal cortex after a PRh-only lesion. In the panels to the right of the lettered sections, the PPRh cortices are $2 \times$ the magnification of the original. 


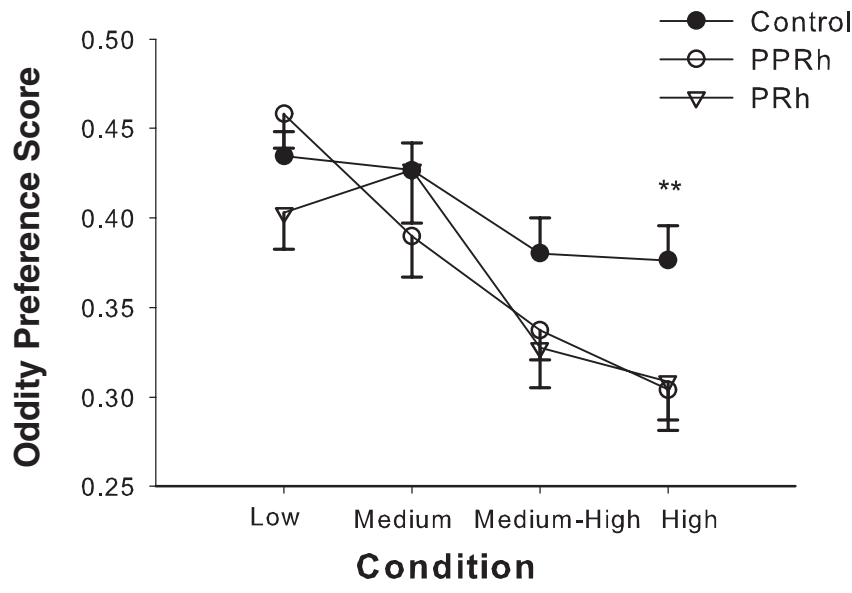

Figure 8. Spontaneous oddity preference performance by control, PPRh, and PRh groups in experiment 4. Data are presented as 3 min cumulative average oddity preference scores (the exploration of the odd object divided by the total exploration of the odd and identical objects combined) \pm SEM. ${ }^{* *} p<0.05$.

low, medium, or medium-high conditions. However, both PPRh and PRh groups were significantly impaired relative to the control group in the high condition $\left(t_{(22)}=2.53 ; p<0.05\right)$ and $\left(t_{(22)}=2.31 ; p<0.05\right)$, respectively. The difference between both lesion groups and the control group in the medium-high condition came very close to reaching significance $\left(\mathrm{PPRh}, t_{(22)}=1.92\right.$, $p=0.06$; PRh, $\left.t_{(22)}=1.86, p=0.07\right)$.

Although analysis of the odd object preference score revealed a significant group by condition interaction at $3 \mathrm{~min}$, the control group showed significant preference for the odd object in the high condition even after only $2 \min \left(t_{(13)}=2.50 ; p<0.05\right)$ (control, $36.91 \pm 2.33 \mathrm{~s}$ ). Neither lesion group showed a significant odd object preference score for the odd object in the high condition at $2 \mathrm{~min}\left(\mathrm{PRh}, t_{(9)}=-1.76, p>0.05\right.$; PPRh, $t_{(9)}=$ $-0.63, p>0.05)(\mathrm{PRh}, 29.62 \pm 2.07 \mathrm{~s}$; PPRh, $32.80 \pm 1.82 \mathrm{~s})$, and this impairment remained throughout the entire $5 \mathrm{~min}$. Furthermore, it is striking that the control rats showed preference for the odd stimulus after $2 \mathrm{~min}$ in the apparatus, as this corresponds to only $\sim 25 \mathrm{~s}$ of object exploration by the rat $(24.30 \pm 0.07 \mathrm{~s}$; object exploration did not significantly differ between groups).

\section{Discussion}

The present results provide evidence that $\mathrm{PRh}$ has a critical role in the perceptual processing of complex objects, in addition to its well established role in memory. Although perceptual manipulations have been shown to affect performance on complex visual discrimination tasks in monkeys and humans, the effect of such manipulations has not been previously explicitly tested in rats. We have shown that, as predicted and as made explicit by the simulations in experiment $1, \mathrm{PRh}$ lesions can produce performance deficits in spontaneous object recognition and oddity discrimination paradigms when stimuli are perceptually similar, even under zero-delay or simultaneous conditions, in which there are little or no long-term memory demands. These deficits were robust, because the performance of rats with PPRh or PRh damage was no better than chance level when the perceptual demand of the tasks were high.

Levy et al. (2005) and Squire et al. (2006) argue that to determine whether perception is impaired during an objectrecognition task, it is important to test animals using no delay between sample and choice. Therefore, in experiment 2, we examined recognition performance of PPRh-lesioned and control rats in a zero-delay object-recognition task using two different levels of perceptual difficulty. When the novel stimulus was not explicitly constructed to resemble the sample stimulus (low condition), the PPRh group was not impaired and performed similarly to the control group. However, when the novel stimulus was constructed to share features in common with the familiar stimulus in the high condition, the PPRh group was impaired relative to the control group. Therefore, the PPRh group was only impaired when the novel object and the familiar object were perceptually similar, necessitating, in our view, the use of conjunctive representations to facilitate recognition. How exactly these conjunctive representations might facilitate recognition is illustrated by the computational model and simulations presented in experiment 1 .

Although experiment 2 examined recognition performance at zero delay, it might be argued that the PRh impairment was caused by a mnemonic failure rather than a perceptual processing failure. Although there was no delay, the rat had to remember the familiar object from the sample phase during the time of the discrimination (in the choice phase) to recognize that the choice object was novel. For this reason, we devised a novel behavioral paradigm, the simultaneous oddity discrimination task, in which two identical stimuli and an odd stimulus are presented to the rat simultaneously, thereby minimizing any memory component in the task. This task was based on the automated oddity task developed by Buckley et al. (2001), on which monkeys with PRh lesions were impaired. Oddity tasks have also been used to assess perceptual deficits in humans with MTL damage (Lee et al., 2005, 2006a) and have shown that damage including the PRh in humans, as in animals, impairs perceptual discrimination of nonspatial stimuli. In experiment 4 of the present study, we examined the performance of rats with lesions of PPRh or PRh and control rats in the simultaneous oddity discrimination task using four different levels of perceptual similarity. [The stimuli used for experiment 4 were first assessed for subjective perceptual similarity in a two-choice visual discrimination experiment (experiment 3).] Both PPRh and PRh groups were impaired relative to the control group in the high condition of the spontaneous oddity task (experiment 4). Thus, even when stimuli were presented simultaneously, the rats with PRh and PPRh lesions could not discriminate the objects in the high condition. However, when the odd object and the identical objects were not perceptually similar, the performances of both lesion groups were indistinguishable from controls. These results extend the findings from experiment 2. The failure of the PPRh and PRh groups to show preference for the odd object can be viewed as a primarily a perceptual, rather than a mnemonic deficit because all of the information necessary to make the oddity judgment was available to the animal simultaneously. The finding that PRh in the rat has not only mnemonic, but perceptual functions that are similar to those performed in PRh in the primate, introduces the rat as a convenient model for studying the mechanisms of perception within the MTL.

Because the zero-delay recognition and simultaneous oddity experiments did not have an explicit control for difficulty, one might argue that performance of any difficult discrimination might be compromised in the rats with PRh lesions. Previous controls for difficulty in complex visual discriminations (Bussey et al., 2002, 2003; Gilbert and Kesner, 2003), oddity tasks (Buckley et al., 2001), and object recognition (Norman and Eacott, 2004) have used size, colors, shapes, or numbers of stimuli as a control for difficulty and have consistently revealed that simply increasing the difficulty of a task is not sufficient to produce PRh 
impairments. In all of these cases, the critical factor determining impairments in PRh-lesioned rats was the stimulus material, or more accurately, the representations necessary for optimal solution of the task (Bussey and Saksida, 2005).

Previous findings from nonhuman primates indicating perceptual impairments after PRh lesions have been criticized on several grounds. Reports of PRh impairments in perceptual processing (Eacott et al., 1994; Buckley and Gaffan, 1997, 1998) have been discounted by claims that the impairment was caused by inadvertent TE damage (Buffalo et al., 1999; Stark and Squire, 2000; Levy et al., 2005). The PPRh and PRh impairments observed in the present experiments cannot be attributed to TE damage. Furthermore, rats in the PRh group, which were significantly impaired in the oddity task, had no TE damage whatsoever. It could also be argued that the deficits produced in experiments 2 and 4 could additionally be attributed to inadvertent damage to entorhinal cortex. However, this is probably not the case because PRh animals with bilateral or unilateral damage to entorhinal cortex did not perform significantly differently from animals without damage to entorhinal cortex in the simultaneous oddity discrimination task. Furthermore, it has been shown that monkeys with ablations to PRh alone can cause severe deficits in visual object recognition memory whereas ablations to entorhinal cortex alone cause only mild deficits (Meunier et al., 1993).

Buffalo et al. (1999) argued that impairments after PRh lesions in a matching-to-sample object-recognition task tested with a zero delay (Eacott et al., 1994) could have been caused by an impairment in the animals with PRh lesions in the retention of the matching rule. The present study replicates the finding of Eacott et al. (1994), in a spontaneous object-recognition task in which there was no matching (or any other) rule to retain; rats perform the task spontaneously. Thus, the interpretation by Eacott et al. (1994) that the impairments produced by PRh lesions were caused by a perceptual impairment is supported by our present findings.

Other criticisms of previous studies include the suggestion that because the oddity task used by others such as Buckley et al. (2001) and Lee et al. (2005, 2006a) involved reward and/or repeated presentation of stimuli, learning across trials may occur in these paradigms and, therefore, deficits on these tasks may be interpreted as impairments in learning (Levy et al., 2005; Shrager et al., 2006; Squire et al., 2006). However, because the spontaneous oddity task used in the present study is a nonrewarded, single-trial paradigm, these criticisms do not apply to the present study.

It might be argued, however, that it does take time for the oddity preference to be expressed in the present paradigm; however, analysis indicates this takes $<25$ s of total object exploration. Furthermore, it is to be expected that it takes some time for object representations to develop and "tune" before difficult perceptual discriminations can effectively be made. Indeed, this is the basic mechanism at work in the model used to generate the simulations in experiment 1 . This development and tuning of representations for perceptual discrimination is often referred to as "perceptual learning," and is thought to be an example of nondeclarative memory (Squire and Zola-Morgan, 1991; Schacter et al., 1993). Our view is that perceptual learning can indeed occur in PRh. Evidence suggests it also does in the hippocampus (Graham et al., 2006).

In summary, the present study shows that $\mathrm{PRh}$ lesions in rats can produce impairments in object recognition and oddity discriminations when items are perceptually similar, even under conditions of zero-delay or simultaneous stimulus presentation, in which there is little or no long-term memory demand. This series of experiments therefore supports the idea that PRh does not contribute solely to long-term declarative mnemonic processing, but has a role in both perception and memory. Thus, the results raise the possibility that, in general, the program of attempting to map psychological concepts such as "perception" and "memory" onto anatomically segregated modules in the brain may not be the best way to understand brain organization (Gaffan, 2002; Bussey and Saksida, 2005). However, when we attempt to describe, at a more mechanistic level, the putative functions of brain regions, it is still important to strive to relate that description to our more everyday understanding of our psychological lives, hence the continued use of terms like perception and memory, even in the present article. Moreover, we will always want to relate our understanding to the way in which neuropsychological patients and their families describe their problems, which is inevitably in psychological terms such as perception and memory (Bussey, 2004). Thus, we may eventually need to embrace a new level of understanding in which psychological terminology is used but only with the understanding that it is appropriate at an abstract level only, whereas a more comprehensive, accurate, and useful account of certain brain functions may be found at a lower, neural network level.

\section{References}

Aggleton JP, Keen S, Warburton EC, Bussey TJ (1997) Extensive cytotoxic lesions involving both the rhinal cortices and area TE impair recognition but spare spatial alternation in the rat. Brain Res Bull 43:279-287.

Brown MW, Aggleton JP (2001) Recognition memory: what are the roles of the perirhinal cortex and hippocampus? Nat Rev Neurosci 2:51-61.

Buckley MJ, Gaffan D (1997) Impairment of visual object-discrimination learning after perirhinal cortex ablation. Behav Neurosci 111:467-475.

Buckley MJ, Gaffan D (1998) Perirhinal cortex ablation impairs visual object identification. J Neurosci 18:2268-2275.

Buckley MJ, Booth MC, Rolls ET, Gaffan D (2001) Selective perceptual impairments after perirhinal cortex ablation. J Neurosci 21:9824-9836.

Buffalo EA, Reber PJ, Squire LR (1998) The human perirhinal cortex and recognition memory. Hippocampus 8:330-339.

Buffalo EA, Ramus SJ, Clark RE, Teng E, Squire LR, Zola SM (1999) Dissociation between the effects of damage to perirhinal cortex and area TE. Learn Mem 6:572-599.

Buffalo EA, Ramus SJ, Squire LR, Zola SM (2000) Perception and recognition memory in monkeys following lesions of area TE and perirhinal cortex. Learn Mem 7:375-382.

Burwell RD (2001) Borders and cytoarchitecture of the perirhinal and postrhinal cortices in the rat. J Comp Neurol 437:17-41.

Bussey TJ (2004) Multiple memory systems: fact or fiction? Q J Exp Psychol 57:89-94.

Bussey TJ, Saksida LM (2002) The organization of visual object representations: a connectionist model of effects of lesions in perirhinal cortex. Eur J Neurosci 15:355-364.

Bussey TJ, Saksida LM (2005) Object memory and perception in the medial temporal lobe: an alternative approach. Curr Opin Neurobiol 15:730-737.

Bussey TJ, Saksida LM, Murray EA (2002) Perirhinal cortex resolves feature ambiguity in complex visual discriminations. Eur J Neurosci 15:365-374.

Bussey TJ, Saksida LM, Murray EA (2003) Impairments in visual discrimination after perirhinal cortex lesions: testing "declarative" vs. "perceptual-mnemonic" views of perirhinal cortex function. Eur J Neurosci 17:649-660.

Cowell R, Bussey T, Saksida L (2006) Why does brain damage impair memory? A connectionist model of object recognition memory in perirhinal cortex. J Neurosci 26:12186-12197.

Dix SL, Aggleton JP (1999) Extending the spontaneous preference test of recognition: evidence of object-location and object-context recognition. Behav Brain Res 99:191-200.

Eacott MJ, Gaffan D, Murray EA (1994) Preserved recognition memory for small sets, and impaired stimulus identification for large sets, following rhinal cortex ablations in monkeys. Eur J Neurosci 6:1466-1478. 
Ennaceur A, Delacour J (1988) A new one-trial test for neurobiological studies of memory in rats. I: Behavioral data. Behav Brain Res 31:47-59.

Forwood SE, Winters BD, Bussey TJ (2005) Hippocampal lesions that abolish spatial maze performance spare object recognition memory at delays of up to 48 hours. Hippocampus 15:347-355.

Gaffan D (2002) Against memory systems. Philos Trans R Soc Lond B Biol Sci 357:1111-1121.

Gilbert PE, Kesner RP (2003) Recognition memory for complex visual discriminations is influenced by stimulus interference in rodents with perirhinal cortex damage. Learn Mem 10:525-530.

Graham KS, Scahill VL, Hornberger M, Barense MD, Lee AC, Bussey TJ, Saksida LM (2006) Abnormal categorization and perceptual learning in patients with hippocampal damage. J Neurosci 26:7547-7554.

Hampton RR (2005) Monkey perirhinal cortex is critical for visual memory, but not for visual perception: reexamination of the behavioral evidence from monkeys. Q J Exp Psychol B 58:283-299.

Holdstock JS, Gutnikov SA, Gaffan D, Mayes AR (2000) Perceptual and mnemonic matching-to-sample in humans: contributions of the hippocampus, perirhinal, and other medial temporal lobe cortices. Cortex 36:301-322.

Lee AC, Buckley MJ, Pegman SJ, Spiers H, Scahill VL, Gaffan D, Bussey TJ, Davies RR, Kapur N, Hodges JR, Graham KS (2005) Specialization in the medial temporal lobe for processing of objects and scenes. Hippocampus 15:782-797.

Lee AC, Buckley MJ, Gaffan D, Emery T, Hodges JR, Graham KS (2006a) Differentiating the roles of the hippocampus and perirhinal cortex in processes beyond long-term declarative memory: a double dissociation in dementia. J Neurosci 26:5198-5203.

Lee AC, Bandelow S, Schwarzbauer C, Henson RN, Graham KS (2006b) Perirhinal cortex activity during visual object discrimination: an eventrelated fMRI study. NeuroImage 33:362-373.

Levy DA, Shrager Y, Squire LR (2005) Intact visual discrimination of complex and feature-ambiguous stimuli in the absence of perirhinal cortex. Learn Mem 12:61-66.

Meunier M, Bachevalier J, Mishkin M, Murray EA (1993) Effects on visual recognition of combined and separate ablations of the entorhinal and perirhinal cortex in rhesus monkeys. J Neurosci 13:5418-5432.

Mumby DG (2001) Perspectives on object-recognition memory following hippocampal damage: lessons from studies in rats. Behav Brain Res 127:159-181.

Mumby DG, Pinel JP (1994) Rhinal cortex lesions and object recognition in rats. Behav Neurosci 108:11-18.
Mumby DG, Glenn MJ, Nesbitt C, Kyriazis DA (2002) Dissociation in retrograde memory for object discriminations and object recognition in rats with perirhinal cortex damage. Behav Brain Res 132:215-226.

Murray EA, Bussey TJ (1999) Perceptual-mnemonic functions of the perirhinal cortex. Trends Cogn Sci 3:142-151.

Norman G, Eacott MJ (2004) Impaired object recognition with increasing levels of feature ambiguity in rats with perirhinal cortex lesions. Behav Brain Res 148:79-91.

Norman KA, O’Reilly RC (2003) Modeling hippocampal and neocortical contributions to recognition memory: a complementary-learningsystems approach. Psychol Rev 110:611-646.

Paxinos G, Watson C (1997) The rat brain in stereotaxic coordinates. New York: Academic.

Pihlajamaki M, Tanila H, Kononen M, Hanninen T, Hamalainen A, Soininen H, Aronen HJ (2004) Visual presentation of novel objects and new spatial arrangements of objects differentially activates the medial temporal lobe subareas in humans. Eur J Neurosci 19:1939-1949.

Schacter DL, Chiu CY, Ochsner KN (1993) Implicit memory: a selective review. Annu Rev Neurosci 16:159-182.

Shrager Y, Gold JJ, Hopkins RO, Squire LR (2006) Intact visual perception in memory-impaired patients with medial temporal lobe lesions. J Neurosci 26:2235-2240.

Squire LR, Zola-Morgan S (1991) The medial temporal lobe memory system. Science 253:1380-1386

Squire LR, Shrager Y, Levy D (2006) Lack of evidence for a role of medial temporal lobe structures in visual perception. Learn Mem 13:106-107.

Stark CE, Squire LR (2000) Intact visual perceptual discrimination in humans in the absence of perirhinal cortex. Learn Mem 7:273-278.

Suzuki WA, Zola-Morgan S, Squire LR, Amaral DG (1993) Lesions of the perirhinal and parahippocampal cortices in the monkey produce longlasting memory impairment in the visual and tactual modalities. J Neurosci 13:2430-2451.

Winters BD, Forwood SE, Cowell RA, Saksida LM, Bussey TJ (2004) Double dissociation between the effects of peri-postrhinal cortex and hippocampal lesions on tests of object recognition and spatial memory: heterogeneity of function within the temporal lobe. J Neurosci 24: 5901-5908.

Xiang J, Brown MW (1998) Differential neuronal encoding of novelty, familiarity, and recency in regions of the anterior temporal lobe. Neuropharmacology 37:657-676 